\title{
Haptic perception of gravitational and inertial mass
}

\author{
WOUTER M. Bergmann TIEST AND ASTRID M. L. KAPPERS \\ Utrecht University, Utrecht, The Netherlands
}

\begin{abstract}
Mass can be perceived in different ways: statically, through gravitational cues; dynamically, through inertial cues; or a combination of both. This article investigates the relationship between these modes of perception. In three different experiments, subjects matched masses that were held statically in the hand to masses that were either accelerated or decelerated. Accelerated masses were perceived to be smaller than masses of equal physical magnitude held statically by a factor of 2 . However, decelerated masses were matched veridically to masses held statically. This difference remained present when contact duration was made very short. This shows that the shift in perceived mass is not the result of differences in the information available, but of differences in the mode of perception (active acceleration vs. passive deceleration). It is hypothesized that this is due to a suppression of the perception of applied force in active touch.
\end{abstract}

Mass is a property of objects in the physical world that manifests itself in two different ways. First of all, two masses exert a gravitational force upon each other that is proportional to the magnitude of either mass. This causes an object to have a certain weight on Earth, and this weight can be measured by measuring the gravitational force. Second, the mass causes an object to have inertia, which is a resistance against changes in velocity. The magnitude of this inertia is also proportional to the mass and can be measured by measuring the force that is needed for a certain acceleration of the object. The mass that causes weight is called gravitational mass, and the mass that causes inertia is called inertial mass. For a given object, these two masses turn out to be of identical magnitude. In Newtonian mechanics, this could be ascribed only to a happy coincidence. However, Einstein showed in his theory of relativity that this was no coincidence at all and that gravitational and inertial mass necessarily had to be the same. This became known as the Einstein equivalence principle, which states that there is no way to distinguish between the force that we experience when we are accelerated (e.g., in a rocket ship in space) and the force that we experience from gravity (e.g., standing still on Earth). The question we would like to answer in this article is whether the Einstein equivalence principle holds for haptically perceived mass. That is, can people correctly match mass perceived through the force of gravity (weight) to mass perceived through inertia?

When judging the weight of an object, the typical exploratory procedure that people use is called unsupported holding, which consists of lifting the object and moving it up and down (Lederman \& Klatzky, 1987). This movement helps one to gain a more precise impression of the object's weight (Brodie \& Ross, 1985). This is assumed to be because, with the involvement of movement, the object's inertia can be perceived in addition to its weight. So, strictly speaking, moving an object about does not help with weight discrimination but gives an impression of the object's inertia, which is related to its mass, which in turn is related to its weight. Apparently, people can use these relationships to aid them in weight discrimination. How well they can use inertial cues to discriminate mass, as compared with a combination of gravitational and inertial cues, has been investigated under weightlessness during parabolic flights and in space. During the brief periods of weightlessness provided by parabolic flights, thresholds for mass discrimination were higher by a factor of about 2 than with normal gravity (Ross \& Reschke, 1982). During the prolonged period of weightlessness of the first Spacelab mission, these thresholds were higher by a factor of about 1.8 than with normal gravity (Ross, Brodie, \& Benson, 1984, 1986). This is not surprising, because under conditions of weightlessness, less information about the mass is available. With normal gravity, both gravitational and inertial cues are available. Under weightlessness, only inertial cues are available. These experiments do show that people are able to use inertial cues to discriminate mass but that they are not as accurate as in a situation in which they also have gravitational cues available. However, the discrimination experiments do not tell us whether the perception of mass based on inertial cues is consistent with the perception of mass based on gravitational cues or a combination of inertial and gravitational cues. Ross and Reschke also performed a magnitude estimation experiment under weightlessness. However, in this experiment, the subjects had to relate their responses to a standard that

W. M. Bergmann Tiest, w.m.bergmanntiest@uu.nl 
also was weightless. So, if they perceived the magnitude of the test masses differently, they would also perceive the magnitude of the standard differently, and the magnitude estimates that they provided still could not give an indication of whether the masses were perceived in the same way or not. Recognizing this problem, the authors performed a small additional experiment in which they had 7 subjects compare a 50 -g ball under weightlessness (during parabolic flight) with another 50-g ball under normal gravity (during normal flight). The weightless ball was found to be lighter by a factor of about 2 than the ball handled under normal gravity. This suggests that masses are not perceived correctly on the basis of inertial cues.

This factor of 2 between the magnitudes of perceived mass with and without gravity is not related to the factor of 2 between the discrimination thresholds with and without gravity. There is no reason why a change in perceived mass would lead to a similar change in the Weber fraction for discrimination. So, the reason for the factor of about 2 found by Ross and Reschke (1982) is a bit of a mystery. There is little to go on, since in their small experiment, only one mass was used. It is unknown whether the same factor would hold for other masses. Furthermore, the experiment did not make a complete separation between inertial and gravitational cues, because in the normal gravity situation, subjects were instructed to shake the stimulus. This provided them with inertial cues in addition to the gravitational cues. Lastly, because the estimation of the gravitational mass had to be done during normal flight, whereas the estimation of the inertial mass had to be done during the execution of a parabola, there was quite a long time between the two estimations (on the order of minutes). This might have caused deviations related to memory effects.

Not only is this factor of 2 an intriguing mystery, but also there is a fundamental interest in studying the way in which the different cues are combined to form a percept of mass. Weight can be perceived statically (without movement), whereas inertia has to be perceived dynamically (with movement). Therefore, studying the roles of these cues can tell us something about the way static and dynamic touch are combined. To shed some light on the mystery of the factor of 2 , we compared the haptic perception of gravitational and inertial mass, using a wider range of masses, an almost complete separation of the cues, and a short time between the perceptions of the gravitational and inertial masses. The inertial cues were separated from the gravitational cues by suspending the mass from a high point. Subjects could experience the inertia of the mass by pushing it sideways. This arrangement was similar to the one used by Ross and Brodie (1987), who measured discrimination thresholds for inertial mass and a combination of inertial and gravitational masses. They used brass and aluminium cylindrical canisters suspended on strings. In this situation, the gravitational force exerted on them was counteracted by the tension in the strings. Thus, when a canister was moved from side to side, mainly the inertial force was experienced. In the present experiments, a similar concept was used, but there were some important differences. In the present experiments, a high fixation point of $2.85 \mathrm{~m}$ was used, as compared with the $0.43 \mathrm{~m}$ in Ross and Brodie. This ensured that, in the present experiments, the angle between the wires and the vertical remained small during the push $\left(<10^{\circ}\right)$, so that only a negligible portion of the pushing force was counteracted by gravitational force. In this way, pushing the mass provided almost exclusively inertial cues. Furthermore, Ross and Brodie asked subjects to grasp the masses and swing them from side to side. Although they were instructed not to, it cannot be ruled out that they lifted the masses a little bit in the process, thus receiving gravitational cues. This problem can be excluded when masses are pushed with the flat hand, as was the case in the present experiments. Lastly, in the weight condition in Ross and Brodie, in which masses had to be lifted, the subjects made vertical oscillatory movements, thus receiving both gravitational and inertial cues. This confound was prevented in the present experiments by asking subjects to hold their hand still while supporting the mass. In short, care was taken to attain the best separation of gravitational and inertial masses possible in a laboratory on earth.

In the present set of experiments, we performed a full investigation of the relationship between inertial mass and gravitational mass in perception. We investigated the roles of the magnitude of the mass itself, the contact duration, and the contact method. In Experiments 1 and 2, the inertial mass was accelerated actively by the subject, whereas in Experiment 3, the inertial mass was decelerated passively.

\section{EXPERIMENT 1 Active Acceleration}

In the first experiment, perception of gravitational mass was compared with perception of inertial mass, using active acceleration over an extended trajectory.

\section{Method}

We compared the haptic perception of gravitational mass and inertial mass, using a guided matching procedure. This meant that the subjects were repeatedly presented with two masses to compare, one perceived through gravitational forces and the other through inertial forces. From these two masses, they had to select the greater one. The perceived difference between the two became progressively smaller until they were felt to be of equal magnitude. This procedure was chosen in favor of a magnitude estimation task because, in this procedure, only a binary answer was required of the subject (the first or the second object had greater mass). This is more intuitive and demands a lower cognitive load than does having to come up with a number to describe a sensation.

Subjects. Eight university students (6 of them female) participated in the experiment. They ranged in age from 18 to 24 years. One other participant was left out of the analysis because, after debriefing, it turned out that she had calculated the ratio between the forces involved in pushing and supporting and had based her judgment on that. In doing so, she performed the task in a different way, resulting in highly deviating data. All the subjects were strongly right-handed, according to Coren's (1993) test. Before the experiment, they signed a declaration of informed consent. They were paid for their time.

Materials. The masses consisted of aluminium containers $(55 \times$ $55 \times 55 \mathrm{~mm}$ ) in which a varying number of brass plates (maximum, 18) could be inserted. The plates were held in place by ridges on the inside of the walls. An illustration is shown in Figure 1. The 


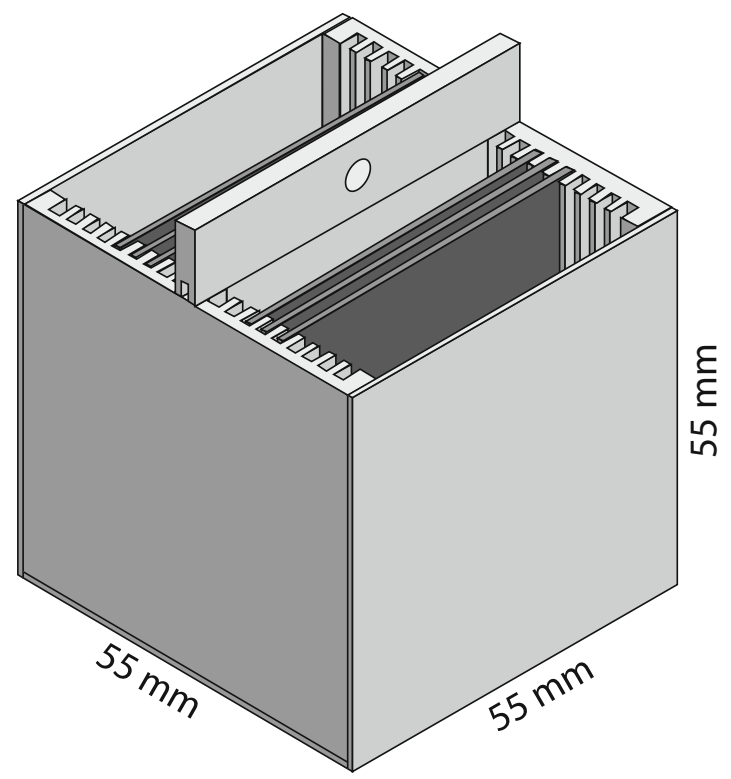

Figure 1. Illustration of the containers used.

mass of an empty container was $102 \mathrm{~g}$, and that of each brass plate was $33.3 \mathrm{~g}$, resulting in a mass range of $0.10-0.70 \mathrm{~kg}$. The containers were carefully designed so that the center of mass was exactly in the center of the container, independently of the number of brass plates inserted (as long as these were placed symmetrically). The mass and torque of the bottom was compensated for by a bar at the top of the container, which contained a hole for hanging. The containers were cubic to ensure that the contact area was the same for pushing from the side and supporting from below. With an even number of plates, 10 different test masses could be created.

The mass to be perceived through inertial cues was suspended from a high point, $2.85 \mathrm{~m}$ above table level, with a pair of ultrahigh molecular weight polyethylene (UHMWPE) wires (cross section, $0.25 \mathrm{~mm}^{2}$ ). These came together at a small metal hook to which the container could be attached. The double wires ensured that the mass could swing but not rotate easily. The total mass of the hook and wires was $\sim 2 \mathrm{~g}$. A stand was provided so that the subject could position his or her hand correctly for pushing horizontally against the mass. A schematic illustration of the setup is shown in Figure 2.

Procedure. The subject was asked to repeatedly compare a mass accelerated sideways with a mass supported from below or vice versa and to indicate which was the greater mass. The ordering of the two ways of perception within each trial was random. When the experimenter gave the instruction to support, the subject placed his or her elbow on the table and held out his or her hand horizontally, about $15 \mathrm{~cm}$ above the table, with the palm facing upward. The experimenter then placed the container on the fingers and removed it after $3 \mathrm{sec}$. The subject had to keep his or her hand still while supporting the mass. The container was placed and removed slowly so as to minimize movement on the part of the subject. He or she was able to adjust the upward force while keeping the hand in place. Over the course of the experiment, the experimenter checked whether the hand had been kept still. When the experimenter gave the instruction to push, the subject placed his or her hand with the back against the stand that was fixed to the table and the palm facing left. The experimenter then placed a hanging container against the fingers. The stand was placed so that the hanging container just touched the subject's hand but did not exert a noticeable force. At the experimenter's signal, the subject was to accelerate the mass sideways, keeping in contact with it for about $50 \mathrm{~cm}$. The mass was then left to fly and was caught by the experimenter. In both pushing and supporting, the mass was placed against the fingers of the outstretched hand, in order to make these two ways of perceiving mass as similar as possible in terms of forces and contact area.

During the push, the inertial force is given by $F_{i}=m a$, where $m$ is the mass and $a$ is the acceleration. The gravitational force in the direction of pushing is $F_{g}=m g \sin \alpha$, where $g$ is the gravitational acceleration $\left(9.8 \mathrm{~m} / \mathrm{sec}^{2}\right)$ and $\alpha$ is the angle between the wires and the vertical. This angle is given by $\sin \alpha=x / h$, where $x$ is the horizontal displacement and $h=2.85 \mathrm{~m}$ is the length of the wires. Thus, the ratio between gravitational and inertial forces during the push is given by $F_{g} / F_{i}=g x / h a$. On the basis of motion tracker measurements (see below), from which the displacement and the acceleration could be derived, this ratio was measured to be, on average, $0.06 \pm 0.02$. That is, the contribution of the gravitational force to the pushing force was about $6 \%$, and about $94 \%$ was inertial force. This demonstrates that the arrangement with the suspended mass came fairly close to providing purely inertial cues.

Before the experiment began, the task was made clear by using an example of having to set a loaded wheelbarrow in motion and having to lift its contents. The subjects were asked to use this kind of daily life experience to make the comparison between the masses. After that, they were blindfolded and performed a practice trial to make sure that they understood the process. Then the experiment proper began.

On each trial, a test stimulus was always paired with one of four reference masses. The order in which test and reference masses were presented within a trial was randomized. After presentation of both stimuli, the subject had to respond as to which was the greater mass by saying "the first" or "the second." The reference masses were gravitational masses of 0.17 and $0.30 \mathrm{~kg}$ and inertial masses of 0.44 and $0.70 \mathrm{~kg}$. These masses were chosen in such a way that the matching gravitational and inertial masses would span most of the available range, on the basis of pilot experiments, with a safe margin for individual differences. The test masses were determined by a one-up/one-down staircase procedure. This means that when the test mass was perceived to be greater (smaller) than the reference mass, the next test mass to be paired with that same reference mass would be one step smaller (greater). The step size was the mass of two brass plates, $66.6 \mathrm{~g}$. In this way, the perceived difference between test and reference masses became progressively smaller until the point of subjective equality (PSE) was reached. For each reference mass,

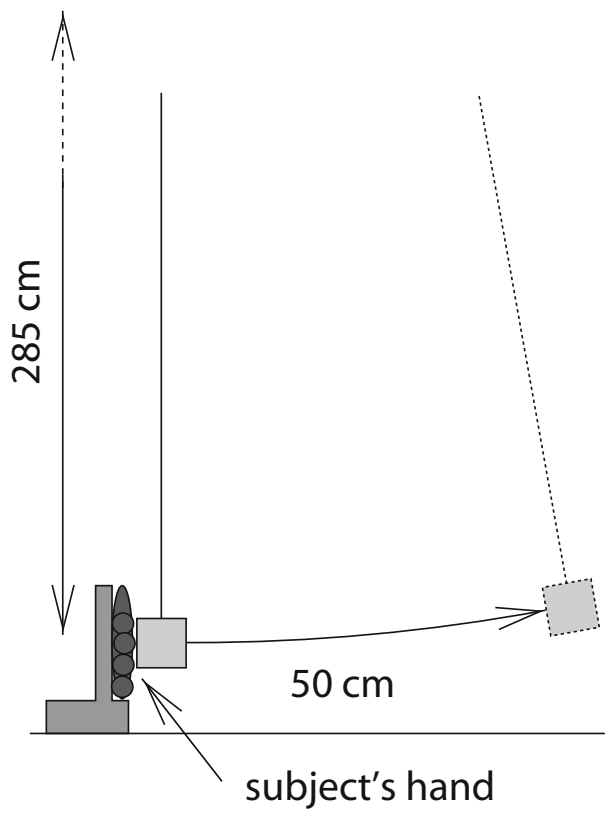

Figure 2. Schematic illustration of the setup for perceiving inertial mass. 


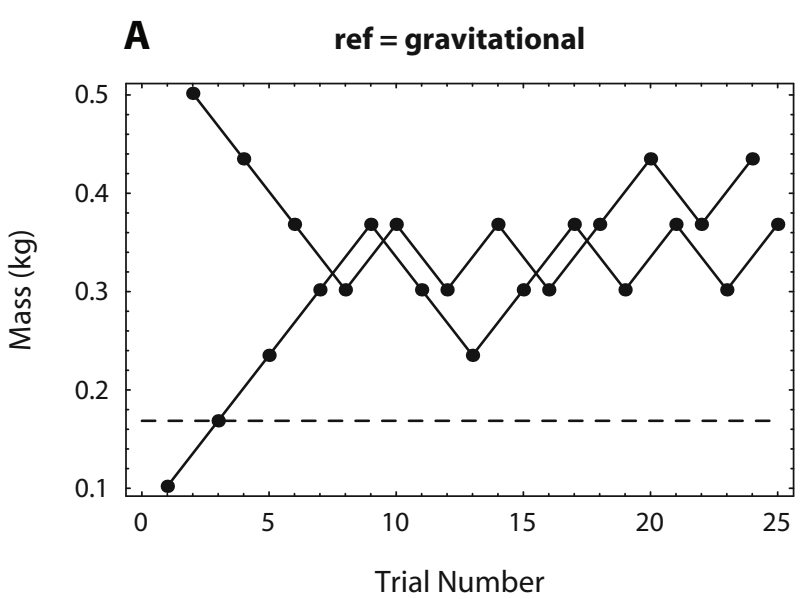

B ref = gravitational

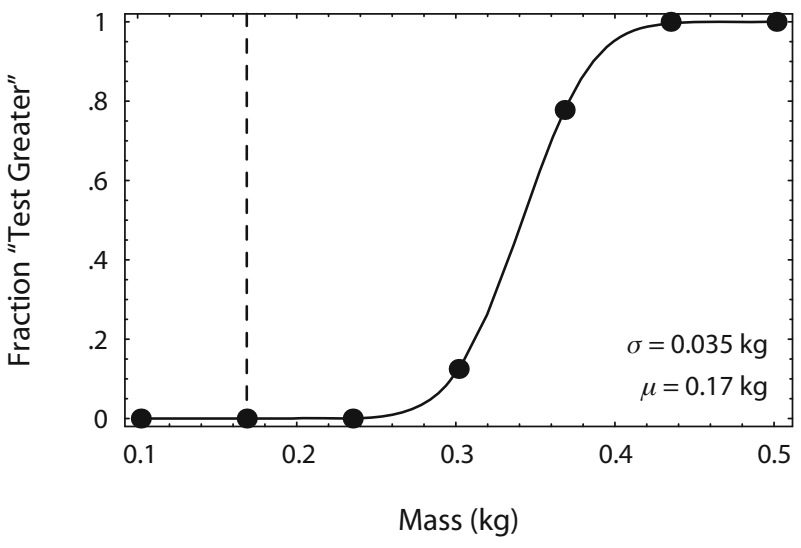

Figure 3. (A) Example of two interleaved staircase procedures. The dots indicate the inertial test mass for each trial. The dashed line indicates the gravitational reference mass. (B) Psychometric curve derived from the staircase data: the fraction of times that the test mass was perceived to be greater than the reference mass, plotted as a function of the magnitude of the test mass. The dots are the data points, and the solid line is a fit to the data. The dashed line indicates the position of the reference. The fitted values of the steepness parameter $\sigma$ and the bias $\mu$ are indicated.

there were two staircases: one starting from above and one from below. The total of eight staircases were presented interleaved. For each reference mass, 25 trials were performed. The total of 100 trials took about 50 min per subject.

Analysis. For each subject and each reference mass, the fraction of times that the test mass was perceived to be greater than the reference mass was plotted as a function of the magnitude of the test mass. An example is shown in Figure 3. To accurately determine the $50 \%$ point (PSE) of the psychometric curve, a cumulative Gaussian function of the form

$$
f(m)=\frac{1}{2}+\frac{1}{2} \operatorname{erf}\left(\frac{m-m_{\mathrm{ref}}-\mu}{\sqrt{2} \sigma}\right)
$$

was fitted to the data. Here, $m_{\text {ref }}$ is the reference mass, and $\mu$ is the difference between the gravitational and inertial masses that are perceived to be equal; $\sigma$ is a parameter that determines the steepness of the curve and is interpreted as a discrimination threshold. In this case, the physical meaning of this parameter is not readily clear, but the magnitude of $\mu$ can be compared with it in order to assess its significance.

\section{Results}

All the subjects included in the analysis showed large biases toward perceiving a gravitational mass as greater than an inertial mass of the same magnitude. Averaged over 8 subjects, the biases for all four reference masses were significantly different from zero (two-tailed $t$ tests, $p<.0001$ for all four tests). The two gravitational reference masses were matched to inertial test masses that were greater by factors of 2.4 and 2.1 , respectively. The two inertial reference masses were matched to gravitational test masses that were smaller by factors of 2.2 and 1.9 , respectively. These values are all close to a factor of 2 , but they are not independent of the mass. This can be seen in Figure 4, in which the inertial mass is plotted against the gravitational mass to which it is perceptually equivalent. The straight line that is fitted to the averages $\left(m_{i}=\right.$ $\left.1.6 m_{g}+0.12 \mathrm{~kg}, R^{2}=.98\right)$ does not pass through the origin. Therefore, the ratio between perceptually equivalent inertial and gravitational masses is not constant. It is around 2 but decreases with increasing mass. A linear

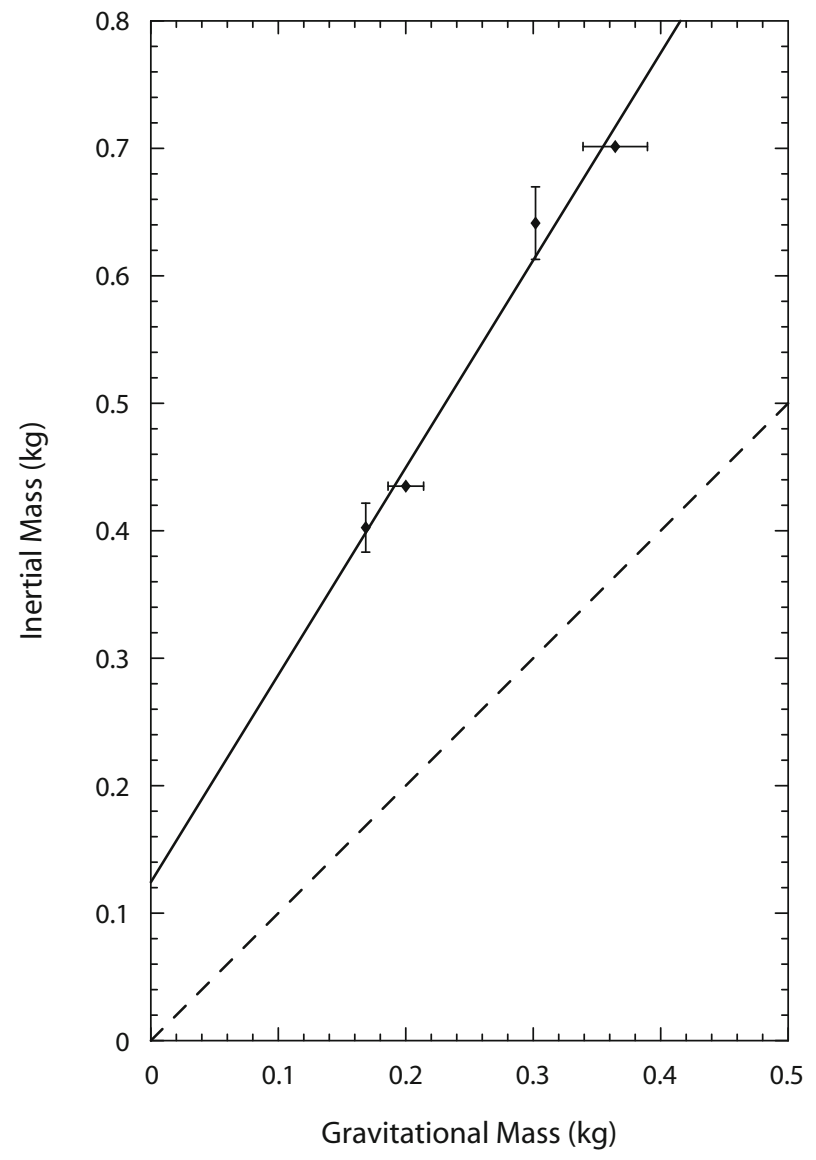

Figure 4. Inertial mass plotted against the gravitational mass to which it was perceptually equal in Experiment 1, averaged over 8 subjects (black diamonds). The error bars indicate the standard errors of the sample means. The solid line is a straight line fitted to the averages minimizing the vertical distance between the points and the line. The dashed line indicates a situation of no perceptual bias, in which gravitational and inertial mass feel the same. 
regression on this ratio as a function of gravitational mass for the data of all the subjects shows that the slope is significantly different from zero $(p<.0001)$ : The greater the mass, the smaller the ratio between inertial and gravitational masses.

In order to be able to assess between-subjects variability, the ratios of perceptually equal inertial and gravitational masses are plotted in Figure 5. For all the subjects, the ratios averaged over reference mass are higher than 1.8. Note that, strictly speaking, the ratios should not be averaged over reference mass, since they are not independent of reference mass, but the averages are used here for clarity.

\section{Discussion}

The main result of this experiment is that in a situation in which gravitational and inertial mass are separated but are perceived in quick succession, inertial mass has to be greater than gravitational mass by a factor of about 2 in order to feel the same. It is as if the subject thinks that he or she is standing on a planet with half the Earth's density, taking the reduced gravity into account. Furthermore, this factor decreases with increasing mass, from 2.4 at $0.17 \mathrm{~kg}$ to 1.9 at $0.36 \mathrm{~kg}$. Despite this variation, within the range measured in this experiment, the numbers are much greater than 1 . The factor of around 2 between gravitational and inertial masses that feel equal is compatible with the finding in Ross and Reschke (1982) that a mass perceived only through inertial cues feels about half as heavy. On the basis of the present finding that the factor seems to depend on the mass, we would have expected a greater factor for the relatively small mass of $0.05 \mathrm{~kg}$ used by Ross and Reschke. That this was not the case might have been due to the fact that in their normal-gravity condition, inertial cues were also available. This mixing of cues might have led to a smaller difference than would be expected on the basis of the present data. This smaller difference means that inertial cues influence the perception of mass under conditions of normal gravity.

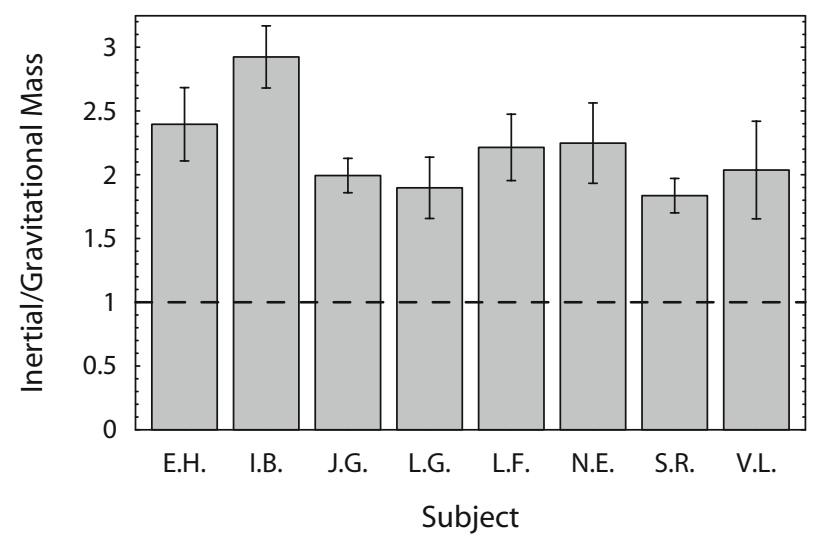

Figure 5. Ratio of perceptually equal inertial and gravitational masses averaged over four reference masses for the different subjects in Experiment 1. The error bars indicate the standard deviations of the averages. The dashed line indicates a ratio of 1 , meaning no perceptual bias.
This leads to the following question: If inertial cues contribute to the perception of mass when gravitational cues are also available, and inertial and gravitational mass are perceived differently (as was demonstrated by the present experiment), how does combining these cues make the perception of mass more accurate? This increased accuracy is demonstrated by lower Weber fractions for the combination of gravitational and inertial cues, as compared with just inertial cues (Brodie \& Ross, 1985; Ross \& Brodie, 1987; Ross et al., 1984, 1986; Ross \& Reschke, 1982). It is true that more information is available when both inertial and gravitational cues are available, but if this information is contradictory (i.e., inertial cues indicate $0.18 \mathrm{~kg}$, gravitational cues indicate $0.40 \mathrm{~kg}$ ), how is the information combined to form a correct percept? In a discrimination experiment with two stimuli, four items of information are available: the perceived weight of the first stimulus, the perceived inertia of the first stimulus, the perceived weight of the second stimulus, and the perceived inertia of the second stimulus. Due to the difference in mass perception based on weight and inertia, comparing these four items with each other would lead to an ambiguous result. It could be that weight and inertia, although perceived simultaneously, are processed separately. A decision about which stimulus is heavier would then be made separately for weight and inertia, and only then would these decisions be combined (using appropriate weight factors) to form a final decision. In this way, advantage is taken of the multiple sources of information available, without the problems related to incompatible mass estimates.

However, when subjects are asked to directly compare weight and inertia, as in the present experiment, this process breaks down, as is demonstrated by the observed biases. But where do these biases come from? The forces involved in the two ways of perceiving mass are quite different: During supporting, the force is equal to the weight of the object, whereas during pushing, the force depends on the acceleration the object is given. Although the subjects were free to use any acceleration they desired, in general this acceleration would be smaller than the gravitational acceleration. Therefore, the associated force would also be smaller than the object's weight. Even though the subjects knew this from earlier experience and should have corrected for it, it could be that the inequality of forces influenced them in their perception. The forces applied during pushing were derived from measured accelerations (see below). The average ratio between the weight of the object and the average applied force during the push was $1.6 \pm 0.2$. This is not quite as high as the ratio between perceptually equal inertial and gravitational mass, but it could contribute to the effect. So, although the experimenter asked the subjects to compare the masses, they may have let the comparison of forces play a role in their decision. The smaller force involved in pushing could have led them to perceive the pushed object as having a smaller mass. The observed biases could be partly explained if we assume that subjects pay attention to the forces they apply, instead of the masses they apply them to. 
Besides the inequality of the forces involved, there is another difference in the two ways of perceiving mass. During supporting, perception is static, and no movements are made. During pushing, perception is very dynamic (changes over time), and fairly large movements are made. It could be that these differences played a role in the observed biases. To check for this, Experiment 2 was performed.

\section{EXPERIMENT 2 Short-Duration Acceleration}

This experiment was similar to Experiment 1, with the notable difference that in the present experiment, the movements during the perception of inertial mass were kept very small, with only a very brief contact duration.

\section{Method}

In this experiment, gravitational mass resting on the hand was matched to inertial mass accelerated through a brief slap instead of a long push.

Subjects. Again, 8 experimentally naive subjects ( 5 of them female), who had not participated in Experiment 1, were recruited. Their ages ranged from 17 to 25 years, and they were all righthanded.

Materials. The same materials as those in Experiment 1 were used. The stand for the subject's hand was placed about $5 \mathrm{~cm}$ from the hanging mass, so as to give the subject a little room to move his or her hand before hitting the mass.

Procedure. The procedure was almost identical to that in Experiment 1 . However, when accelerating a mass, the subject was asked to use a quick, short motion to slap the hanging container and to keep the contact duration to a minimum. The subject placed his or her hand against the stand, with about $3 \mathrm{~cm}$ distance between the fingers and the hanging mass. At the experimenter's signal, the subject made a short movement with the wrist, slapping the mass with the fingers. On the basis of motion tracker measurements (see below), the contact time was $0.07 \pm 0.02 \mathrm{sec}$, and in that time, the fingers moved $7 \pm 3 \mathrm{~cm}$. Note that, whereas in Experiment 1 there was a small gravitational component in the push situation, the cues were completely separated in the present experiment. The subjects were asked to recall the experience of hitting a tennis ball with a racquet and to compare this with their memory of holding a tennis ball in their hand. In this way, they were to match the gravitationally perceived mass to the inertially perceived mass and vice versa. The same four reference masses were used as in Experiment 1, and for each, 25 trials were performed. The experiment took about $50 \mathrm{~min}$ per subject.

Analysis. The analysis was identical to that in Experiment 1: By fitting a psychometric curve to the data, the bias between the two ways of perceiving mass was determined.

\section{Results}

Averaged over subjects, the biases found were significantly different from zero (two-tailed $t$ tests, $p \leq .00028$ ). The two gravitational reference masses were matched to inertial masses by a factor of 1.9 and 2.0 greater, respectively. Conversely, the inertial reference masses were matched to gravitational masses by a factor of 1.8 smaller. The results are depicted graphically in Figure 6. A straight line $\left(m_{i}=\right.$ $1.8 m_{g}+0.090 \mathrm{~kg}, R^{2}=.98$ ) was fitted to the averages. The constant term was not significantly different from zero ( $p=.88)$, meaning that the line passes through the origin. This indicates that the ratio of perceptually equal inertial

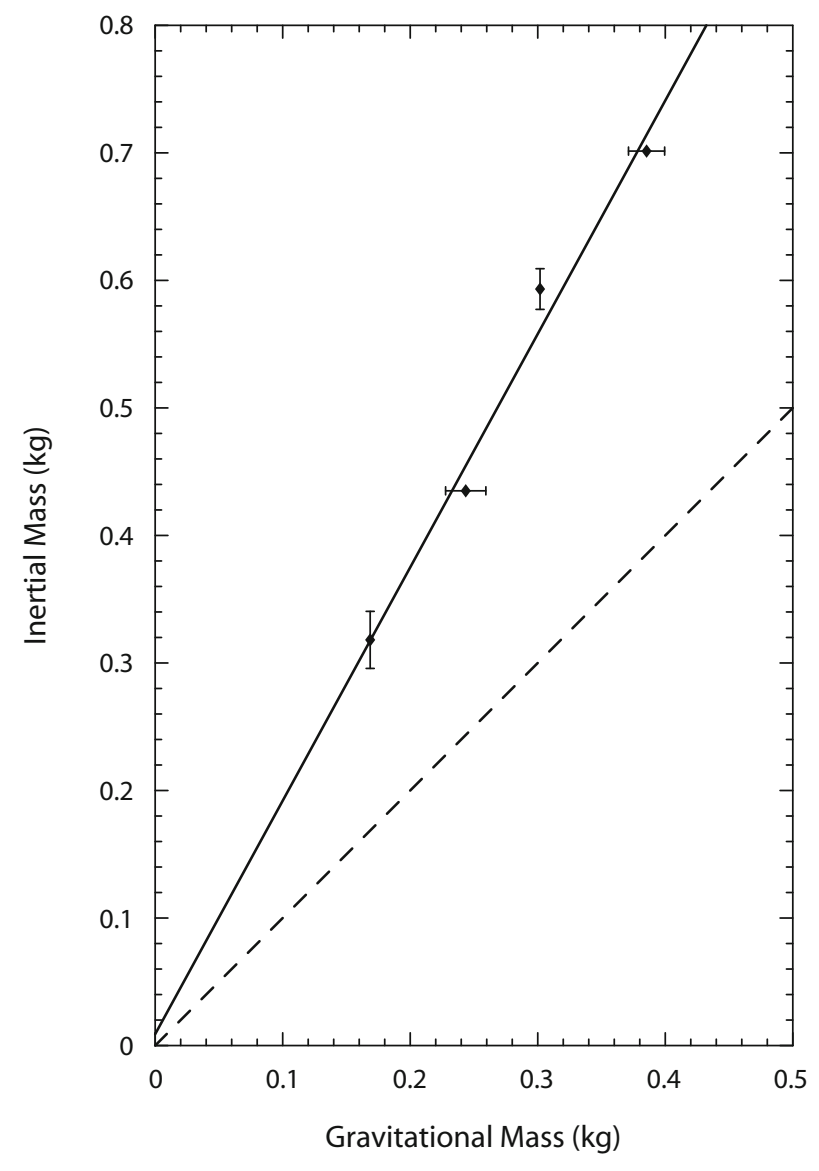

Figure 6. Inertial mass plotted against the gravitational mass to which it was perceptually equal in Experiment 2, averaged over 8 subjects (black diamonds). The error bars indicate the standard errors of the sample means. The solid line is a straight line fitted to the averages. The dashed line indicates a situation of no perceptual bias.

mass and gravitational mass does not depend on the magnitude of the mass, which is confirmed by the fact that the slope of this ratio as a function of gravitational mass is not significantly different from zero $(p=.18)$.

In order to be able to assess the between-subjects variability, the ratios of perceptually equal inertial and gravitational masses are plotted in Figure 7. For all the subjects, the ratios averaged over reference mass are higher than 1.5 .

\section{Discussion}

It appears also to be the case that when inertial mass is accelerated through a quick slap, it is judged to be much smaller than gravitational mass. The ratios in this experiment with the small movements seem to be somewhat smaller than those in Experiment 1 with the large movements. However, this difference is not statistically significant [MANOVA on the ratios for the four reference masses in the two experiments, $F(4,11)=1.7, p=.22]$. This means that with the small movements, the subjects were equally biased in matching gravitational mass and inertial mass. So, the large biases in Experiment 1 were not due to 


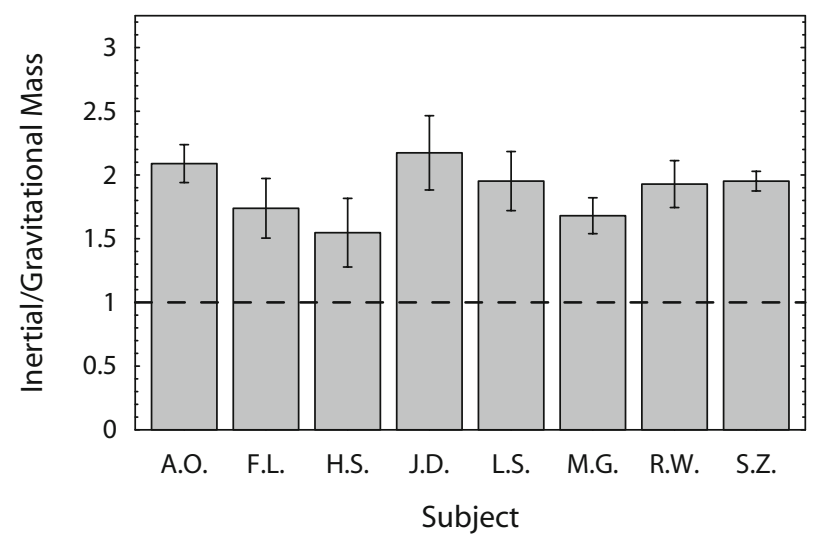

Figure 7. Ratio of perceptually equal inertial and gravitational masses averaged over four reference masses for the different subjects in Experiment 2. The error bars indicate the standard deviations of the averages. The dashed line indicates a ratio of 1 , meaning no perceptual bias.

the difference in the scale of the movements between supporting and pushing: When a mass was accelerated with a very small movement, an equally large bias was found. The contact duration was much shorter than during pushing in the previous experiment $(0.07 \pm 0.02 \mathrm{sec}$, instead of $0.35 \pm 0.04 \mathrm{sec})$, but the masses were observed to be given a velocity that was not much smaller $(1.4 \pm 0.2 \mathrm{~m} / \mathrm{sec}$, instead of $2.2 \pm 0.2 \mathrm{~m} / \mathrm{sec}$ ). Therefore, although of shorter duration, the forces applied were much higher during the slap than during pushing. In fact, the average ratio between the weight of the object and the average applied force during the slap was $0.5 \pm 0.1$. If the subjects were using the applied force as a cue, as was suggested in the Discussion section of Experiment 1, we would expect the higher force applied during the slap to lead to the inertial mass being perceived as larger, whereas the opposite was observed. Thus, an influence of force cannot be an explanation for the observed bias in the present experiment.

Although the overall difference in ratios between Experiments 1 and 2 was not significant, there were differences between the experiments for the individual reference masses. Contrary to Experiment 1, the ratios found in the present experiment did not seem to depend on the magnitude of the mass. To test whether this difference was significant, the intercepts of the straight lines fitted to the data of the individual subjects were compared. These were significantly different between Experiments 1 and 2 (two-tailed $t$ test, $t=2.3, p=.037$ ). This suggests that there may have been multiple components in the biases found: a constant relative bias that was always present and a mass-dependent bias that was present only when the contact duration was longer and that was added to the constant relative bias.

\section{EXPERIMENT 3}

\section{Passive Deceleration}

Acceleration is not the only way in which inertia can be perceived. Also, inertia manifests itself in deceleration (braking). In principle, the same information is available, regardless of whether a mass is accelerated or decelerated. Therefore, on the basis of the findings from Experiments 1 and 2, one would also expect a large bias when inertia is perceived through deceleration instead of acceleration. On the other hand, there is also a difference between accelerating and decelerating a mass in whether perception is active or passive. In Experiments 1 and 2, gravitational mass was perceived passively (the mass was placed on the subject's hand by the experimenter), whereas inertial mass was perceived actively (the subject was in control of his or her movements while accelerating the mass). If this distinction were responsible for the biases, they might disappear when inertial mass is perceived passively. To investigate the bias between gravitational mass and inertial mass perceived through passive deceleration, a third experiment was performed.

\section{Method}

In this experiment, again, mass perceived through inertial cues had to be matched to mass perceived through gravitational cues and vice versa. Only this time, the inertial cues were provided not by accelerating a stationary mass, but by decelerating (stopping) a moving mass.

Subjects. Once again, 8 university students (6 of them female) participated in this experiment, none of whom had participated in Experiment 1 or 2 . They ranged in age from 19 to 24 years. One of them was strongly left-handed, according to Coren's (1993) test. The subjects used their preferred hand for the experiment.

Materials. The same materials as those in Experiments 1 and 2 were used. Instead of the stand for the subject to rest the hand on, a small pole was fixed to the table, which the subject could use to position the hand correctly. The pole had a ring around it to indicate the correct height. There was another pole to mark a point $50 \mathrm{~cm}$ from the subject's hand, where the suspended mass could be released to swing toward the hand.

Procedure. Again, the task was to compare gravitationally and inertially perceived masses. The procedure for perceiving gravitational mass was the same as that in the previous experiments: static supporting. For perceiving inertial mass, the blindfolded subject placed the flat hand with the palm facing sideways, with the ends of the fingers against the small pole. The pole was present only to mark the correct location of the hand, and the subject could move the hand freely. The experimenter held the mass $50 \mathrm{~cm}$ away and told the subject to prepare for stopping it. The mass was then released and bumped into the subject's hand. To prevent the subject from using the sound of the impact as a cue, he or she wore earplugs. Neglecting air drag, ${ }^{1}$ the velocity of all masses at the moment of impact was $v=\sqrt{2 g h[1-\cos (l / h)]} \approx 0.93 \mathrm{~m} / \mathrm{sec}$, where $g$ is the gravitational acceleration $\left(9.8 \mathrm{~m} / \mathrm{sec}^{2}\right), h$ is the height of the fixation point $(2.85 \mathrm{~m})$, and $l$ is the length of the trajectory $(0.50 \mathrm{~m})$. The subject was allowed to move the hand a little with the mass while stopping it. The subject kept his/her hand quite still, but especially for the larger masses, it did move a little bit on impact. The posture of the hand did not change. Also, in this experiment, no gravitational component was present during the perception of inertial mass. After perceiving masses in both ways, the subject had to say which one was the greater. The same interleaved staircase procedure was used. Again, there were two gravitational reference masses $(0.17$ and $0.37 \mathrm{~kg})$ and two inertial reference masses $(0.37$ and $0.63 \mathrm{~kg})$. The mass of $0.37 \mathrm{~kg}$ is in the center of the stimulus range and was chosen because, in pilot experiments, subjects did not appear to have a bias either way for this way of perceiving mass. The other two masses were chosen toward either end of the stimulus range, just in case the results of the real experiment deviated from those of the pilots. For each of the four references, 25 trials were performed. The total of 100 trials per subject took about $50 \mathrm{~min}$. 


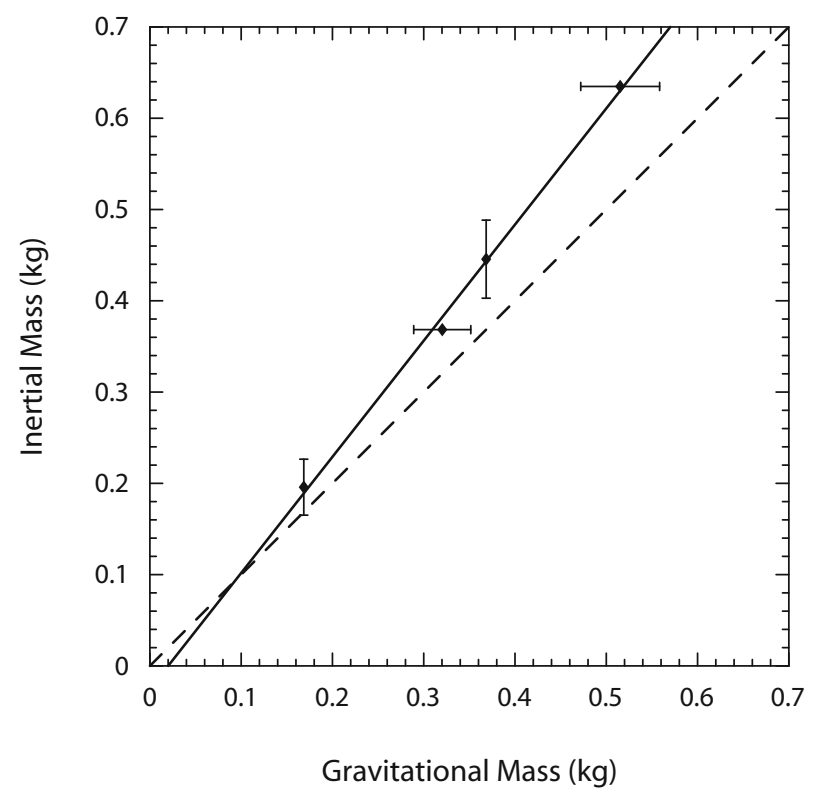

Figure 8. Inertial mass plotted against the gravitational mass to which it was perceptually equal in Experiment 3, averaged over 8 subjects (black diamonds). The error bars indicate the standard errors of the sample means. The solid line is a straight line fitted to the averages. The dashed line indicates a situation of no perceptual bias.

Analysis. The analysis was the same as that in Experiments 1 and 2 .

\section{Results}

When masses decelerated passively were compared with masses held statically on the hand, only small or negligible biases were found. On average, the two gravitational masses were matched to inertial masses by a factor of 1.2 greater. The two inertial masses also were matched to gravitational masses by a factor of 1.2 smaller. Only for the largest inertial mass was the bias significantly different from zero (two-tailed $t$ test, $p=.028$ ); the other three biases were not significant $(p \geq .11)$. The results are represented graphically in Figure 8. As can be seen in the figure, the deviation from veridical matching (indicated by the dashed line) was much smaller than in the previous experiment. That is, when the subjects had to match a mass resting on the hand to a moving mass that was stopped, they made only small errors, whereas when they had to match a mass resting on the hand to a mass that was accelerated, they made very large errors.

The straight line fitted to the averages $\left(m_{i}=1.3 m_{g}-\right.$ $0.03 \mathrm{~kg}, R^{2}=.997$ ) comes very close to the origin; the constant term is not significantly different from zero $(p=.27)$. This suggests that the ratio of perceptually equal inertial and gravitational masses is more or less constant for different masses. Indeed, a linear regression on this ratio as a function of gravitational mass for the data of all the subjects shows a slope that is not significantly different from zero $(p=.22)$.

The ratio of inertial mass and gravitational mass is approximately the same for the different masses in Ex- periment 3. However, this does not hold for the different subjects. In Figure 9, this ratio, averaged over the four reference masses, is shown for the different subjects. It can be seen that whereas most subjects have a ratio close to 1,2 subjects (F.B. and, especially, F.D.) have ratios that are much larger. This means that most of the subjects actually had no or very small biases and were able to match gravitational and inertial mass correctly by passively decelerating a moving mass.

\section{Discussion}

When decelerated mass is compared with gravitational mass, the decelerated mass has to be greater than the gravitational mass by a factor of 1.2 , on average, to feel equivalent. The fact that this number deviates from 1 (no perceptual bias) appears to have been due to 1 or 2 subjects. So, most subjects are able to make a correct match between a mass supported statically and a mass bumping into the hand with a speed of around $1 \mathrm{~m} / \mathrm{sec}$, even though the pattern of forces is very different: The gravitational force is constant during the $3 \mathrm{sec}$ that the mass rests on the hand, whereas the impact force has the character of a peak with a very short duration. It could be that experience with catching objects, where a moving object is first stopped (whereby its inertial mass can be perceived) and then held (whereby its gravitational mass can be perceived), has taught subjects to correctly match these different patterns of forces.

When comparing this experiment with the previous one, the differences in observed biases are striking. In the two experiments, the same information is available: the weight and the inertia of the masses. The lengths of time during which the information is available ( $3 \mathrm{sec}$ for gravitational mass, $<0.1 \mathrm{sec}$ for inertial mass) are also comparable. Still, on the basis of this information, a very different percept is formed, depending on whether the masses are accelerated or decelerated. Apparently, whether inertial mass is accelerated actively or decelerated passively is crucial for whether its magnitude can be perceived correctly.

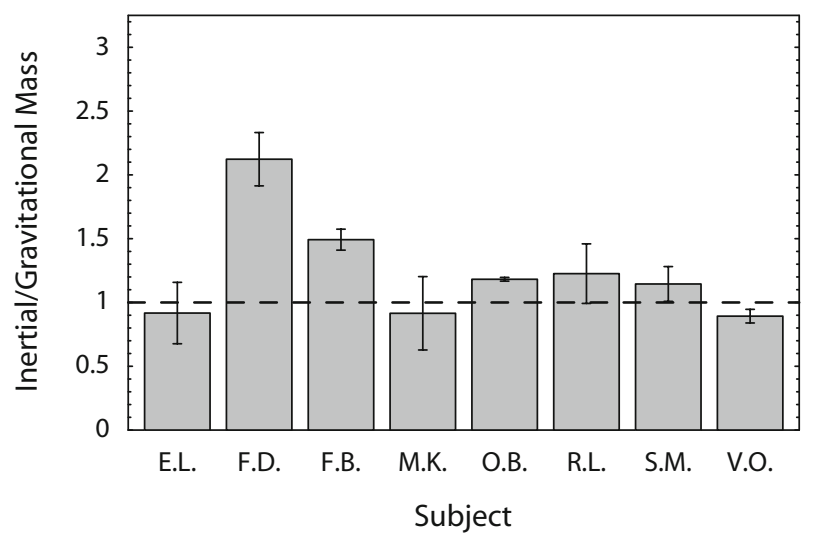

Figure 9. Ratio of perceptually equal inertial and gravitational masses averaged over four reference masses for the different subjects in Experiment 3. The error bars indicate the standard deviations of the averages. The dashed line indicates a ratio of 1 , meaning no perceptual bias. 


\section{MOTION TRACKER MEASUREMENTS}

In order to gain more insight into the movements made when inertial mass was perceived in different ways, measurements were performed using a NDI Optotrak Certus 3-D motion tracker. This device is able to track the position of tiny infrared light-emitting diodes (LEDs) over time with an accuracy of about $0.2 \mathrm{~mm}$. These LEDs can be attached to moving objects. We used one LED on the tip of the middle finger of a subject and one LED on the center of the hanging container. Positions were sampled at a rate of $100 \mathrm{~Hz}$. Movement tracks were recorded for all 10 test masses, ranging from 0.10 to $0.70 \mathrm{~kg}$, for pushing, brief slap, and stopping. Recordings lasted for a few seconds. From the 3-D position data, the velocity in the direction of movement was calculated by taking the derivative of the $x$, $y$, and $z$ components and adding these quadratically. From the velocity data, acceleration was calculated by taking the second derivative. By multiplication with the mass, forces were calculated from the acceleration data. Contact time could be calculated by determining the time during which the acceleration was above a threshold of $1 \mathrm{~m} / \mathrm{sec}^{2}$ (pushing and brief slap) or below $-5 \mathrm{~m} / \mathrm{sec}^{2}$ (stopping). On the basis of the times of the beginning and the end of contact, the distance traveled while in contact with the mass was calculated. The impulse (amount of momentum transferred during contact) is equal to the time-integrated force. The work (amount of mechanical energy transferred during contact) is equal to the distance-integrated force. The relevant data are given in Table 1. Contact times were about $0.3 \mathrm{sec}$ for pushing, about $0.06 \mathrm{sec}$ for brief slap, and about $0.04 \mathrm{sec}$ for stopping. This indicates that the contact times for the last two ways of perceiving inertial mass are quite comparable and much shorter than that for the first method. For pushing, acceleration did not depend on mass, meaning that the subject automatically adjusted the applied force to the different masses. This becomes very apparent in the strong correlation of the applied force with the mass $\left(R^{2}=.94\right)$. For the other two ways of perceiving inertial mass, the correlation is not so strong, and the acceleration (deceleration in the case of stopping) is found to depend on the mass. Apparently, during the brief contact, there is not enough time to fully adjust the force to the mass. This manifests itself also in the contact times and traveled distances that depend on mass for brief slap and stopping. For stopping, the work done during the de- celeration shows an almost perfect correlation with mass $\left(R^{2}=.98\right)$. This is not surprising, since the velocity at the moment of impact is the same for all masses. Therefore, the kinetic energy depends linearly on mass, and the work done while stopping the mass is equal to its kinetic energy. For pushing, the traveled distance is about $30 \mathrm{~cm}$, quite a bit less than the instructed $50 \mathrm{~cm}$. This is a clear sign of the haptic underestimation of distance.

It appears that for pushing, the subject based his or her perception of inertial mass solely on the force (or impulse or work; the three are closely related) necessary to keep the acceleration the same for all masses. With the other ways of perceiving inertial mass, he or she could have used a combination of contact time, traveled distance, acceleration, and force.

\section{GENERAL DISCUSSION AND CONCLUSIONS}

Presented here are three very similar experiments with very different outcomes. When actively accelerated mass is compared with statically supported mass, the former has to be larger than the latter by a factor of about 2 to feel equivalent. This confirms the finding of Ross and Reschke (1982) that a mass of $50 \mathrm{~g}$ is judged to be half as heavy under conditions of weightlessness. In their article, Ross and Reschke speculated that the misperception during weightlessness could be due to incomplete adaptation to the situation. The present findings do not support this speculation: In our experiments, there was no adaptation involved, but still the inertial mass was misperceived. The present experiments also expand the earlier finding by showing how the ratio of perceived inertial mass and gravitational mass behaves over a range of masses and by showing that active acceleration is required for this effect to occur. When passively decelerated mass is compared with statically supported mass, they are matched veridically in most cases. In both situations (active and passive), the same information is available: gravitational and inertial forces. Therefore, the large difference between the situations is very surprising. Perhaps it can be related to subjects' previous experience. As was remarked before, the force patterns of stopping and supporting an object might have been linked to each other through experience with catching objects. However, the same would hold for throwing objects and the relationship between accelerating and supporting objects.

Table 1

Relevant Parameters Derived From the Motion Tracker Measurements

\begin{tabular}{lccc}
\hline \multicolumn{1}{c}{ Parameter } & Pushing (Experiment 1) & Brief Slap (Experiment 2) & Stopping (Experiment 3) \\
\hline Contact time $(\mathrm{sec})$ & $0.35 \pm 0.04$ & $0.09 m+0.03\left(R^{2}=.64\right)$ & $0.04 m+0.026\left(R^{2}=.47\right)$ \\
Traveled distance $(\mathrm{m})$ & $0.30 \pm 0.05$ & $0.01 m+0.08\left(R^{2}=.44\right)$ & $0.022 m+0.017\left(R^{2}=.64\right)$ \\
Mean velocity $(\mathrm{m} / \mathrm{sec})$ & $0.87 \pm 0.07$ & $0.7 \pm 0.2$ & $0.64 \pm 0.05$ \\
Mean acceleration $\left(\mathrm{m} / \mathrm{sec}^{2}\right)$ & $6.3 \pm 0.7$ & $-27 m+35\left(R^{2}=.44\right)$ & $-29 m+36\left(R^{2}=.49\right)$ \\
Mean force $(\mathrm{N})$ & $6.4 m\left(R^{2}=.94\right)$ & $12 m+4\left(R^{2}=.55\right)$ & $16 m+2\left(R^{2}=.85\right)$ \\
Impulse $(\mathrm{Ns})$ & $2.06 m\left(R^{2}=.98\right)$ & $1.4 m\left(R^{2}=.88\right)$ & $0.62 m\left(R^{2}=.93\right)$ \\
Work $(\mathrm{J})$ & $2.2 m+0.02\left(R^{2}=.93\right)$ & $1.0 m\left(R^{2}=.68\right)$ & $0.41 m\left(R^{2}=.98\right)$ \\
\hline
\end{tabular}

Note-For quantities that are independent of mass, the average value and standard deviation are given. For quantities that do depend on mass (slopes significantly different from zero at $p<.05$ level), a straight line fit in terms of the mass $m$ (in kilograms) and an $R^{2}$ value are given. 
When mass is accelerated by means of a quick slap, the ratio between an inertial mass and a gravitational mass that are perceived to be equal does not depend on the mass. This seems to be the main difference with the situation in which mass is accelerated through a longer push.

Although the three experiments were very similar, there were a few differences that might explain the results. Since the perception of gravitational mass was identical in all three experiments, we must focus on differences in the perception of inertial mass.

First of all, there was a difference in the movements that were made. In the first experiment, fairly large movements were made that included the hand, wrist, arm, and shoulder. In the second experiment, these movements were very small and mostly involved only the hand. It has been shown that the perceived magnitude of a force depends on the muscle group producing the force (Jones, 2003). This would predict a difference in perceived mass between supporting and brief slap (Experiment 2), but not so much between supporting and pushing (Experiment 1). However, large biases were observed in both cases, so the involvement of the arm and shoulder does not seem to play a key role in these biases. If we assume that the bias in the first experiment arose because the pushed mass was perceived to be lighter than it actually was, we can explain this by hypothesizing that the distance moved while the mass was accelerated was overestimated. This would be consistent with the motion tracker measurements of traveled distance during pushing. If the subject thinks he or she has moved over a larger distance in the same time interval, he or she will conclude that the acceleration is larger than it actually is. For the same force, this would lead to the perception of a smaller mass. Thus, in order for a pushed mass to feel equivalent to a supported mass, it needs to be larger. In the second experiment, there also was almost no involvement of the limbs, and the movement was limited to the hand and wrist. Still, if the extent of these smaller movements is also overestimated, we should expect biases in the second experiment, as were observed. However, in the third experiment, movements of a similar size were made, but no large biases were observed. Therefore, this explanation of the difference, based on overestimation of movements, cannot hold.

Second, the forces involved in the perception of inertial mass differed among the three experiments. The results of Experiment 1 could be partly explained if we assume that the subjects were judging applied force instead of mass. The force applied when a mass is pushed sideways is indeed smaller than the gravitational force exerted on the supported mass. However, this could not explain the results of Experiments 2 and 3: There, the forces involved in the perception of inertial mass were much higher than the gravitational force. If judgment were based on force in these experiments, too, the inertial mass would be considered larger than the gravitational mass. Instead, a bias in the other direction or no bias was found. Furthermore, the forces involved in perception of inertial masses in Experiments 2 and 3 were of the same order of magnitude, but the inertial masses were perceived very differently in these two experiments. So, differences in force cannot explain the observed biases.
Third, there was a difference in the duration of the inertial forces. In Experiment 1, the inertial force was experienced over the amount of time it took to move the mass about $50 \mathrm{~cm}$, which was observed to be a couple of tenths of a second. In Experiments 2 and 3, the force was experienced only over a very short duration, below $0.1 \mathrm{sec}$. If, for some reason, a short contact duration leads to a more veridical perception, that could explain the difference between Experiments 1 and 3. However, then one would also expect small biases in Experiment 2, which were not observed. Therefore, differences in contact duration cannot explain the differences in bias. With a short duration, as in Experiments 2 and 3 , the ratio of inertial and gravitational masses perceived as equal did not depend on mass. This may be due to the fact that in those cases, the observer did not have time to adjust his or her movement during contact with the mass. A time of about $100 \mathrm{msec}$ is needed to adjust shoulder and elbow movement to unexpected inertial loads (Smeets, Erkelens, \& Denier van der Gon, 1990). It may be that with the prolonged contact in Experiment 1, the observer unconsciously adapted his or her movement to the magnitude of the mass perceived at the start of the contact. This type of adaptation to inertial load has been observed in cat muscle (Partridge, 1967) and humans (Lestienne, 1979). This adaptation could have led to larger inertial masses being perceived differently from smaller inertial masses.

Fourth, the amount and direction of momentum transfer between hand and mass were different. The amount was largest in Experiment 1 and smallest in Experiment 3. Since the inertial masses were perceived to be largest in Experiment 3, it is unlikely that momentum transfer plays a role in the perception of inertial mass. A similar point can be made about the mechanical work done when masses are accelerated or decelerated.

Finally, there is a difference in whether perception is active or passive. In the first and second experiments, the subjects were actively in control of the movement. In the third experiment, they had to wait passively until impact was made. There has been some research into the difference between active and passive touch (Symmons, Richardson, \& Wuillemin, 2004). In this article, a large number of studies comparing the accuracy of active and passive touch were reviewed, and it turned out that there has not been a clear preference for either. Therefore, it cannot be concluded that passive touch is more accurate. Loomis and Lederman (1984) even questioned the utility of the distinction between active and passive touch and placed more emphasis on the type of information available, cutaneous or kinesthetic. The authors reviewed a number of studies and concluded that when the availability of afferent kinesthetic information is controlled for, there is little difference in performance between active and passive touch. Regarding weight perception, however, an advantage was found for active lifting, as compared with lifting through the tonic vibratory reflex (Brodie \& Ross, 1984). When a weight is lifted by applying a vibratory stimulus to the tendon of the biceps muscle, no efferent signals are involved, and only afferent kinesthetic information is available. The authors concluded that both afferent and efferent signals 
contribute to weight discrimination. This would indicate that active perception of weight should be more accurate than passive, but the accuracy of perception is not necessarily related to perceptual biases, as was observed in Experiments 1 and 2. These biases may be the result of misperception of limb movement. In this respect, differences between active and passive perception have been found (Gritsenko, Krouchev, \& Kalaska, 2007). In this study, the subjects had to match movements of the elbow joint that were perceived actively or passively. However, from the results, it seems that overestimation of limb position is greater in the case of passive movements, which is incongruent with the present findings, where the largest biases were found with active movements. The difference in active and passive perception of inertial mass could be related to the difference in active and passive perception of force, as was most graphically demonstrated by Shergill, Bays, Frith, and Wolpert (2003). In that experiment, it was shown that force originating from an external cause (being pushed) is always perceived as greater than self-generated force (pushing) of an equal physical magnitude. This explains why physical conflicts tend to escalate quickly. It might also explain why inertial mass is perceived to be larger when a moving mass is stopped than when a stationary mass is set into motion. A similar effect has been observed with the perceived intensity of cutaneous perception of electrical stimuli on the index finger with and without movement (Milne, Aniss, Kay, \& Gandevia, 1988). With finger movement, the perceived intensity is reduced by about $10 \%$. This may be attributed to so-called gating of the transmission of somatosensory signals to the primary somatosensory cortex (Chapman, 1994). Motor activity diminishes the perception of cutaneous stimuli by suppressing the transmission of afferent input, both at the level of the dorsal column and higher up the medial lemniscal pathway. This means that cutaneous stimulation will be experienced as less intense during arm or hand movement than without movement. Since the perception of acceleration and deceleration forces in the present experiments was at least partially mediated through the cutaneous channel, this could contribute to the observed difference in inertial mass. Combined with the overestimation of traveled distance that leads to an underestimation of inertial mass, the gating phenomenon could, in part, explain the observed biases.

In conclusion, we have shown that gravitational and inertial mass are perceived quite differently when perception of the inertial mass is active but that they can be matched veridically when perception of the inertial mass is passive. The reason for this distinction between active and passive perception could lie in the suppression of cutaneous stimulation during movements.

\section{AUTHOR NOTE}

This research was supported by a grant from the Netherlands Organisation for Scientific Research (NWO). Correspondence should be addressed to W. M. Bergmann Tiest, Helmholtz Institute, Utrecht University, Padualaan 8, $3584 \mathrm{CH}$ Utrecht, The Netherlands (e-mail: w.m.bergmanntiest@uu.nl).

\section{REFERENCES}

Brodie, E. E., \& Ross, H. E. (1984). Sensorimotor mechanisms in weight discrimination. Perception \& Psychophysics, 36, 477-481.

Brodie, E. E., \& Ross, H. E. (1985). Jiggling a lifted weight does aid discrimination. American Journal of Psychology, 98, 469-471.

Chapman, C. E. (1994). Active versus passive touch: Factors influencing the transmission of somatosensory signals to primary somatosensory cortex. Canadian Journal of Physiology \& Pharmacology, 72, 558-570.

Coren, S. (1993). The left-hander syndrome: The causes and consequences of left-handedness. New York: Vintage Books.

Gritsenko, V., Krouchev, N. I., \& Kalaska, J. F. (2007). Afferent input, efference copy, signal noise, and biases in perception of joint angle during active versus passive elbow movements. Journal of Neurophysiology, 98, 1140-1154.

JoNES, L. A. (2003). Perceptual constancy and the perceived magnitude of muscle forces. Experimental Brain Research, 151, 197-203.

Lederman, S. J., \& KLATZKy, R. L. (1987). Hand movements: A window into haptic object recognition. Cognitive Psychology, 19, 342368

LESTIENNE, F. (1979). Effects of inertial load and velocity on the braking process of voluntary limb movements. Experimental Brain Research, 35, 407-418

Loomis, J. M., \& Lederman, S. J. (1984, November). What utility is there in distinguishing between active and passive touch? Paper presented at the 25th Annual Meeting of the Psychonomic Society, San Antonio, TX.

Milne, R. J., Aniss, A. M., Kay, N. E., \& Gandevia, S. C. (1988). Reduction in perceived intensity of cutaneous stimuli during movement: A quantitative study. Experimental Brain Research, 70, 569-576.

PARTRIDGe, L. D. (1967). Intrinsic feedback factors producing inertial compensation in muscle. Biophysical Journal, 7, 853-863.

Ross, H. E., \& Brodie, E. E. (1987). Weber fractions for weight and mass as a function of stimulus intensity. Quarterly Journal of Experimental Psychology, 39A, 77-88.

Ross, H. E., Brodie, E. E., \& Benson, A. J. (1984). Mass discrimination during prolonged weightlessness. Science, 225, 219-221.

Ross, H. E., Brodie, E. E., \& Benson, A. J. (1986). Mass-discrimination in weightlessness and readaptation to earth's gravity. Experimental Brain Research, 64, 358-366.

Ross, H. E., \& ReschKe, M. F. (1982). Mass estimation and discrimination during brief periods of zero gravity. Perception \& Psychophysics, 31, 429-436.

Shergill, S. S., Bays, P. H., Frith, C. D., \& Wolpert, D. M. (2003). Two eyes for an eye: The neuroscience of force escalation. Science, 301, 187.

Smeets, J. B. J., Erkelens, C. J., \& Denier van der Gon, J. J. (1990). Adjustments of fast goal-directed movements in response to an unexpected inertial load. Experimental Brain Research, 81, 303-312.

Symmons, M., Richardson, B. L., \& Wuillemin, D. B. (2004). Active versus passive touch: Superiority depends more on the task than the mode. In S. Ballesteros Jiménez \& M. A. Heller (Eds.), Touch, blindness, and neuroscience (pp. 179-185). Madrid: Universidad Nacional de Educación a Distancia.

\section{NOTE}

1. When air drag is included in the calculations, the velocity is mass dependent, but the variation in velocity over the range of masses used in this experiment is estimated to be no more than $0.5 \%$, on the basis of a calculated upper limit for the drag force.

(Manuscript received September 8, 2009; revision accepted for publication January 23, 2010.) 